\title{
EFFECTIVE UNIFORM APPROXIMATION BY THE RIEMANN ZETA-FUNCTION
}

\author{
Ramūnas Garunkštis, Antanas LaurinčIKas, \\ Kohji Matsumoto, Jörn Steuding, and Rasa Steuding
}

Dedicated to Prof. Dr. Wolfgang Schwarz on the occasion of his 75th birthday Abstract

We apply an effective multidimensional $\Omega$-result of Voronin in order to obtain effective universality-type theorems for the Riemann zeta-function. We further use this approach to study approximation properties of linear combinations of derivatives of the zeta-function.

\section{History of Voronin's work and related results}

In 1975, Voronin [17] proved a remarkable universality theorem for $\zeta(s)$ which states, roughly speaking, that any non-vanishing analytic function can be approximated uniformly by certain purely imaginary shifts of the zeta-function in the critical strip:

Theorem 1. Let $0<r<\frac{1}{4}$ and suppose that $g(s)$ is a non-vanishing continuous function on the disk $|s| \leq r$ which is analytic in the interior. Then, for any $\epsilon>0$,

$$
\liminf _{T \rightarrow \infty} \frac{1}{T} \operatorname{meas}\left\{\tau \in[0, T]: \max _{|s| \leq r}\left|\zeta\left(s+\frac{3}{4}+i \tau\right)-g(s)\right|<\epsilon\right\}>0 .
$$

It is natural to ask for an effective version of this theorem. Given an admissible function $g$ and some positive $\epsilon$, is it possible to give an upper bound for the first $\tau>0$ such that

$$
\max _{|s| \leq r}\left|\zeta\left(s+\frac{3}{4}+i \tau\right)-g(s)\right|<\epsilon,
$$

or is it possible to give a lower bound for the positive lower density in Theorem 1? This is indeed a very difficult problem and neither the

2000 Mathematics Subject Classification. 11M06, 30E10.

Key words. Riemann zeta-function, universality, uniform approximation. 
known proofs of Voronin's universality Theorem 1 nor its generalizations or extensions are effective. However, there are some remarkable attempts towards a solution of this problem.

First of all, Good [4] combined Voronin's universality Theorem 1 with the work of Montgomery [10] on extreme values of the zeta-function, which enabled him to complement Voronin's qualitative picture with Montgomery's quantitative estimates. His result is rather complicated and a more satisfying statement was recently given by Garunkštis [1] , building on Good's ideas. Garunkštis proved that if $f(s)$ is analytic in $|s| \leq 0.05$ with $\max _{|s| \leq 0.05}|f(s)| \leq 1$, then for any $0<\epsilon<\frac{1}{2}$ there exists a real number $\tau$ with

$$
0 \leq \tau \leq \exp \left(\exp \left(10 \epsilon^{-13}\right)\right)
$$

such that

$$
\max _{|s| \leq 0.0001}\left|\log \zeta\left(s+\frac{3}{4}+i \tau\right)-f(s)\right|<\epsilon,
$$

and further

$$
\begin{gathered}
\liminf _{T \rightarrow \infty} \frac{1}{T} \operatorname{meas}\left\{\tau \in[0, T]: \max _{|s| \leq 0.0001}\left|\log \zeta\left(s+\frac{3}{4}+i \tau\right)-f(s)\right|<\epsilon\right\} \\
\geq \exp \left(-\epsilon^{-13}\right)
\end{gathered}
$$

The original theorem is too complicated to be given here. Laurinčikas [6], [7] found another approach which gives conditional effective results subject to certain assumptions on the speed of convergence of the related limit distribution. However, the rate of convergence of weakly convergent probability measures related to the space of analytic functions is not understood very well. Steuding $[\mathbf{1 2}],[\mathbf{1 3}]$ obtained non-trivial upper bounds for the upper density of universality.

The purpose of the present paper is to give another approach to obtain effective statements on the approximation by $\zeta(s)$. The origin of Voronin's universality Theorem 1 seems to be a density theorem from $[\mathbf{1 5}]$ :

Theorem 2. For any fixed numbers $s_{1}, \ldots, s_{n}$ with $\frac{1}{2}<\operatorname{Re} s_{k}<1$ for $1 \leq k \leq n$ and $s_{k} \neq s_{\ell}$ for $k \neq \ell$, the set

$$
\left\{\left(\zeta\left(s_{1}+i t\right), \ldots, \zeta\left(s_{n}+i t\right)\right): t \in \mathbb{R}\right\}
$$

is dense in $\mathbb{C}^{n}$. Moreover, for any fixed number $s$ in $\frac{1}{2}<\sigma<1$,

$$
\left\{\left(\zeta(s+i \tau), \zeta^{\prime}(s+i \tau), \ldots, \zeta^{(n-1)}(s+i \tau)\right): \tau \in \mathbb{R}\right\}
$$

is dense in $\mathbb{C}^{n}$. 
This theorem is a significant improvement of classic results due to Harald Bohr and his collaborators from the first half of the twentieth century. Of course, Theorem 2 is a simple consequence of Theorem 1, but Theorem 2 was proved earlier than Theorem 1. In [16], Voronin extended his approach in order to prove the joint functional independence of Dirichlet $L$-functions to pairwise inequivalent characters. Actually, this paper may be regarded as forerunner of his universality Theorem 1 . As indicated by Laurinčikas [8], the missing link for obtaining (joint) universality was the absence of a rearrangement theorem for Hilbert spaces which was proved by Pečerskiu [11] on Voronin's request. Garunkštis [2] found a slight modification of Voronin's proof which does not make use of the rearrangement theorem.

Voronin's universality Theorem 1 can be seen as an infinite dimensional analogue of the second part of Theorem 2: the truncated Taylor series of the target function $g(s)$ can be approximated by the truncated Taylor series of a certain shift of zeta. This idea was cultivated by Matsumoto [9] and in the next section we shall combine the idea from [9] with the following Theorem 3 in order to obtain our effective results in the present paper.

In 1988, Voronin [18] proved the following, remarkable effective version of the second part of Theorem 2:

Theorem 3. Let $\sigma \in\left(\frac{1}{2}, 1\right), \epsilon \in(0,1)$ and $\mathbf{b}=\left(b_{0}, b_{1}, \ldots, b_{n-1}\right) \in \mathbb{C}^{n}$ with $\left|b_{0}\right|>\epsilon$ be fixed. Then the system of inequalities

$$
\left|\zeta^{(k)}(\sigma+i t)-b_{k}\right|<\epsilon \quad \text { for } \quad 0 \leq k<n
$$

has a solution $t \in[T, 2 T]$ provided that

$$
T \geq C(n, \sigma) \exp \exp \left(5 A(n, \mathbf{b}, \epsilon)^{\frac{8}{1-\sigma}+\frac{8}{\sigma-1 / 2}}\right),
$$

where $C(n, \sigma)$ is a positive, effectively computable constant, depending only on $n$ and $\sigma$, and

$$
A(n, \mathbf{b}, \epsilon)=|\log | b_{0}||+\left(\frac{\|\mathbf{b}\|}{\epsilon}\right)^{n^{2}}
$$

with $\|\mathbf{b}\|:=\sum_{0 \leq k<n}\left|b_{k}\right|$.

This theorem is also given in the monograph of Karatsuba and Voronin [5]; unfortunately, Voronin called his results $\Omega$-theorems which does not really match the statement. The proof of Theorem 3 is pretty much the same as the one for Theorem 2 with two exceptions which allow the effectiveness of the statement: firstly, the use of systems of linear equations in as many unknowns as equations (solvable by Cramer's rule with 
a solution in an explicit range), which replaces the ineffective rearrangement theorem; secondly, Fourier analysis as substitute for Kronecker's diophantine approximation theorem.

Because of Voronin's untimely death in 1997 we will never know whether this reasoning was Voronin's inspiration for [18]; we believe that Voronin was aware of these applications of his late results from [18] to the effectivity problem in universality and, if he was, we may speculate that he was not completely satisfied.

\section{Effective uniform approximation}

Let $\mathcal{K}=\left\{s \in \mathbb{C}:\left|s-s_{0}\right| \leq r\right\}$, where $r$ is a positive real number and $s_{0}=\sigma_{0}+i t_{0}$ with $\sigma_{0} \in\left(\frac{1}{2}, 1\right)$. Notice that $\mathcal{K}$ is not necessarily included in the strip $\frac{1}{2}<\operatorname{Re} s<1$. Assume that we are given an analytic target function $g: \mathcal{K} \rightarrow \mathbb{C}$. Our main tool is the Taylor series expansion of $g$ :

$$
g(s)=\sum_{k=0}^{\infty} \frac{g^{(k)}\left(s_{0}\right)}{k !}\left(s-s_{0}\right)^{k}
$$

valid for all $s \in \mathcal{K}$. By Cauchy's formula,

$$
g^{(k)}\left(s_{0}\right)=\frac{k !}{2 \pi i} \int_{\left|s-s_{0}\right|=r} \frac{g(s)}{\left(s-s_{0}\right)^{k+1}} \mathrm{~d} s,
$$

where the integral is taken over the circle $\left|s-s_{0}\right|=r$ in counterclockwise direction. Hence,

$$
\left|g^{(k)}\left(s_{0}\right)\right| \leq k ! M r^{-k}
$$

where $M:=\max _{\left|s-s_{0}\right|=r}|g(s)|$. We fix a number $\delta_{0} \in(0,1)$. Then

$$
\left|\frac{g^{(k)}\left(s_{0}\right)}{k !}\left(s-s_{0}\right)^{k}\right| \leq M \delta_{0}^{k} \quad \text { for } \quad\left|s-s_{0}\right| \leq \delta_{0} r .
$$

In order to apply Theorem 3 we suppose that $g\left(s_{0}\right) \neq 0$. Then we can choose a sufficiently small $\epsilon$ satisfying $0<\epsilon<\left|g\left(s_{0}\right)\right|$. There exists a positive integer $n=n\left(\delta_{0}, \epsilon, M\right)$ such that

(3) $\quad \Sigma_{1}:=\left|g(s)-\sum_{0 \leq k<n} \frac{g^{(k)}\left(s_{0}\right)}{k !}\left(s-s_{0}\right)^{k}\right|<\frac{\epsilon}{3} \quad$ for $\quad\left|s-s_{0}\right| \leq \delta_{0} r$. 
Let $0<\delta \leq \delta_{0}$. Then, needless to say, (3) is valid for $\left|s-s_{0}\right| \leq \delta r$. Now we apply Theorem 3 with $b_{k}=g^{(k)}\left(s_{0}\right)$ and $\frac{\epsilon}{3}$ in place of $\epsilon$. Then, for any sufficiently large $T$, there exists $t_{1} \in[T, 2 T]$ such that

$$
\left|\zeta^{(k)}\left(\sigma_{0}+i t_{1}\right)-g^{(k)}\left(s_{0}\right)\right|<\frac{\epsilon}{3} \quad \text { for } \quad 0 \leq k<n ;
$$

the condition with respect to $T$ is

$$
T \geq C\left(n, \sigma_{0}\right) \exp \exp \left(5 A(n, \mathbf{g}, \epsilon / 3)^{\frac{8}{1-\sigma_{0}}+\frac{8}{\sigma_{0}-1 / 2}}\right),
$$

where $\mathbf{g}$ is the vector composed from the coefficients in the Taylor series expansion for $g$. In particular, the first component of $\mathbf{g}$ is $g\left(s_{0}\right)$. Thus,

$$
\begin{aligned}
\Sigma_{2} & :=\left|\sum_{0 \leq k<n} \frac{\zeta^{(k)}\left(\sigma_{0}+i t_{1}\right)}{k !}\left(s-s_{0}\right)^{k}-\sum_{0 \leq k<n} \frac{g^{(k)}\left(s_{0}\right)}{k !}\left(s-s_{0}\right)^{k}\right| \\
& <\frac{\epsilon}{3} \sum_{0 \leq k<n} \frac{(\delta r)^{k}}{k !}<\frac{\epsilon}{3} \exp (\delta r) \text { for }\left|s-s_{0}\right| \leq \delta r .
\end{aligned}
$$

Now let $\tau=t_{1}-t_{0}$; then $\sigma_{0}+i t_{1}=s_{0}+i \tau$. We use the Taylor expansion of $\zeta(s)$ on the disc $\mathcal{K}+i \tau$. For this purpose it is necessary that the pole of $\zeta(s)$ is not included in this disc. This is achieved if

$$
T>r,
$$

which is satisfied if $T$ is sufficiently large. Under this assumption, we have

$$
\zeta(s+i \tau)=\sum_{k=0}^{\infty} \frac{\zeta^{(k)}\left(s_{0}+i \tau\right)}{k !}\left(s-s_{0}\right)^{k}
$$

for $s \in \mathcal{K}$. Put

$$
M(\tau)=\max _{\left|s-s_{0}\right|=r}|\zeta(s+i \tau)| .
$$

Then, again by Cauchy's formula,

$$
\left|\frac{\zeta^{(k)}\left(s_{0}+i \tau\right)}{k !}\left(s-s_{0}\right)^{k}\right| \leq M(\tau) \delta^{k} \quad \text { for } \quad\left|s-s_{0}\right| \leq \delta r .
$$

Hence,

$$
\begin{aligned}
\Sigma_{3} & :=\left|\zeta(s+i \tau)-\sum_{0 \leq k<n} \frac{\zeta^{(k)}\left(s_{0}+i \tau\right)}{k !}\left(s-s_{0}\right)^{k}\right| \\
& =\left|\sum_{k=n}^{\infty} \frac{\zeta^{(k)}\left(s_{0}+i \tau\right)}{k !}\left(s-s_{0}\right)^{k}\right| \leq M(\tau) \frac{\delta^{n}}{1-\delta} \quad \text { for } \quad\left|s-s_{0}\right| \leq \delta r .
\end{aligned}
$$


Putting (3)-(8) together, we find

$$
|\zeta(s+i \tau)-g(s)| \leq \Sigma_{1}+\Sigma_{2}+\Sigma_{3}<\frac{\epsilon}{3}+\frac{\epsilon}{3} \exp (\delta r)+M(\tau) \frac{\delta^{n}}{1-\delta} .
$$

Now choose $\delta>0$ such that

$$
M(\tau) \frac{\delta^{n}}{1-\delta}=\frac{\epsilon}{3}(2-\exp (\delta r))
$$

this is possible since the left hand-side tends to zero as $\delta \rightarrow 0$, while the right hand-side tends to $\frac{\epsilon}{3}>0$, resp. when $\delta \rightarrow 1$ the left-hand side tends to infinity, but the right-hand side remains bounded. We thus have proved

Theorem 4. Let $\sigma_{0} \in\left(\frac{1}{2}, 1\right), g: \mathcal{K} \rightarrow \mathbb{C}$ continuous, $g\left(s_{0}\right) \neq 0$ and analytic for $\left|s-s_{0}\right|<r$. Then, for any $\epsilon \in\left(0,\left|g\left(s_{0}\right)\right|\right)$ there exist real numbers $\tau \in[T, 2 T]$ and $\delta=\delta(\epsilon, g, \tau)>0$ defined by (9) such that

$$
\max _{\left|s-s_{0}\right| \leq \delta r}|\zeta(s+i \tau)-g(s)|<\epsilon
$$

where $T=T\left(g, \epsilon, \sigma_{0}\right)$ has to satisfy (5) and ( 7$)$, and $\delta$ is effectively computable.

Corollary 5. The assertion of Theorem 4 holds even if $g\left(s_{0}\right)=0$, if we replace (5) by (10) below.

Proof: Suppose $g\left(s_{0}\right)=0$. Set $g_{\epsilon}(s)=g(s)+\epsilon / 2$, so that $g_{\epsilon}\left(s_{0}\right) \neq 0$. Since $\epsilon / 3 \in\left(0,\left|g_{\epsilon}\left(s_{0}\right)\right|\right)$, we can apply Theorem 4 to find $\tau \in[T, 2 T]$ and $\delta>0$ for which

$$
\max _{\left|s-s_{0}\right| \leq \delta r}\left|\zeta(s+i \tau)-g_{\epsilon}(s)\right|<\epsilon / 3
$$

holds, and $T$ has to satisfy

$$
T \geq C(n, \sigma) \exp \exp \left(5 A\left(n, \mathbf{g}_{\epsilon}, \epsilon / 3\right)^{\frac{8}{1-\sigma_{0}}+\frac{8}{\sigma_{0}-1 / 2}}\right),
$$

where $\mathbf{g}_{\epsilon}=\left(\epsilon / 2, g^{(1)}\left(s_{0}\right), \ldots, g^{(n-1)}\left(s_{0}\right)\right)$. This clearly implies the assertion of the corollary.

Remark. By the same idea it is possible to show that a slightly modified version of Theorem 3 holds even if $b_{0}=0$. Let $\widetilde{\mathbf{b}}=\left(\epsilon / 2, b_{1}, \ldots, b_{n-1}\right)$. Applying Theorem 3 we find that the system of inequalities $\mid \zeta^{(k)}(\sigma+$ $i t)-\widetilde{b}_{k} \mid<\epsilon / 3$ for $0 \leq k<n$ (where $\widetilde{b}_{0}=\epsilon / 2$ ) has a solution $t \in[T, 2 T]$, provided that

$$
T \geq C(n, \sigma) \exp \exp \left(5 A(n, \widetilde{\mathbf{b}}, \epsilon / 3)^{\frac{8}{1-\sigma_{0}}+\frac{8}{\sigma_{0}-1 / 2}}\right) .
$$


This $t$ is a solution of the system $\left|\zeta^{(k)}(\sigma+i t)-b_{k}\right|<\epsilon$ for $0 \leq k<n$, as desired.

It is remarkable that there is no objection on $g$ to be non-vanishing on $\mathcal{K}$ as in the universality Theorem 1 . Indeed, the statement of Theorem 1 contradicts the Riemann hypothesis if $g$ has a zero in $\mathcal{K}$ (since any such zero would generate via Rouché's theorem many zeros of $\zeta(s+i \tau)$; see $[\mathbf{1 4}, \S 8.1])$. However, it seems that there is an inner mechanism which prevents to obtain an extraordinarily good approximation of the target function. We observe that a small $\epsilon$ leads to a big $T$ in (5) and further that the bigger $T$, the smaller we have to choose $\delta$ via (9). This follows from the fact that the zeta-function is unbounded inside the critical strip and so the quantity $M(\tau)$ is increasing with $T$ to infinity. We illustrate this by an example. Let $s_{0}$ be a complex number with $\frac{1}{2}<\sigma_{0}<1$ and $b>0$. We put $g(s)=b+s-s_{0}$; then $g$ is an entire function with a simple zero in $s_{0}-b$. So we may choose $n=2$ in the above proof of Theorem 4 (and there is no error term coming from (3)). We observe that (9) holds for some sufficiently small $\delta>0$. Now assume that $0<b<\delta r$. Note that $\delta r<\log 2$ holds because the right-hand side of (9) is positive under our choice of $\delta$. If we could choose $\epsilon>0$ such that

$$
\max _{\left|s-s_{0}\right|=\delta r}|\zeta(s+i \tau)-g(s)|<\epsilon<\min _{\left|s-s_{0}\right|=\delta r}|g(s)|=\delta r-b
$$

then we would obtain a disproof of the Riemann hypothesis (if we additionally suppose that $\frac{1}{2}<\sigma_{0}-\delta r$ and $\sigma_{0}+\delta r<1$ in order to have a zero off the critical line). Indeed, the zero of $g(s)$ in $s=s_{0}-b$ lies inside the disk of radius $\delta r$, and so by Rouché's theorem $\zeta(s+i \tau)$ vanishes for some value of $s$ with $\left|s-s_{0}\right|<\delta r$ too. The second inequality of (12) can be fulfilled by choosing $\epsilon$ sufficiently small, comparing with the value of $\delta$. However, in order to fulfill the first inequality of (12), Theorem 4 yields only a large value for $\tau$ which leads to a big $M(\tau)$ so that $\delta$ has to be chosen rather small. Here we see an indirect link between uniform approximation and the order of growth of the zeta-function on vertical lines.

It should be noticed that Gauthier and Clouâtre $[\mathbf{3}]$ obtained a noneffective variant of Theorem 4 ; they proved that every function holomorphic on a compact set $\mathcal{K} \subset \mathbb{C}$ having connected complement can be approximated by translates of Taylor polynomials of the zeta-function. Here $\mathcal{K}$ is not necessarily contained in the right half of the critical strip. Their proof depends on Voronin's theorem. 


\section{Linear combinations}

The approach of the previous section also applies to any derivative $\zeta^{(j)}(s)$ of the zeta-function in place of $\zeta(s)$. Moreover, we can even allow linear combinations.

Theorem 6. Let $\lambda_{0}, \lambda_{1}, \ldots, \lambda_{J}$ be any complex numbers, not all equal to zero, and define

$$
\ell(s)=\sum_{j=0}^{J} \lambda_{j} \zeta^{(j)}(s)
$$

Suppose that $\sigma_{0} \in\left(\frac{1}{2}, 1\right), g: \mathcal{K} \rightarrow \mathbb{C}$ is continuous, $g\left(s_{0}\right) \neq 0$, and analytic for $|s|<r$. Then, for any $\epsilon \in\left(0,\left|g\left(s_{0}\right)\right|\right)$ there exist real numbers $\tau \in[T, 2 T]$ and $\delta=\delta(\epsilon, g, \tau)>0$ defined by (9) such that

$$
\max _{\left|s-s_{0}\right| \leq \delta r}|\ell(s+i \tau)-g(s)|<\epsilon
$$

for all $T>C=C\left(g, \epsilon, \sigma_{0}, \lambda_{0}, \ldots, \lambda_{J}\right)$, where $\delta$ and $C$ are effectively computable positive constants.

Proof: The key idea for the proof of this theorem is also to use Theorem 3. Since the case $J=0$ was already treated by Theorem 4 , we assume $J \geq 1$ in the following proof.

Let $\tau$ be real. Using the local power series expansion

$$
\zeta(s+i \tau)=\sum_{k=0}^{\infty} \frac{1}{k !} \zeta^{(k)}\left(s_{0}+i \tau\right)\left(s-s_{0}\right)^{k},
$$

we find

$$
\ell(s+i \tau)=\sum_{k=0}^{\infty} \frac{1}{k !} \sum_{j=0}^{J} \lambda_{j} \zeta^{(k+j)}\left(s_{0}+i \tau\right)\left(s-s_{0}\right)^{k}
$$

Hence, different from the previous section, we now have to find $\tau$ which is a solution of the system of inequalities

$$
\left|\sum_{j=0}^{J} \lambda_{j} \zeta^{(k+j)}\left(s_{0}+i \tau\right)-g^{(k)}\left(s_{0}\right)\right|<\frac{\epsilon}{3}
$$

for $0 \leq k<n$. For this purpose we solve the system of linear equations

$$
\sum_{j=0}^{J} \lambda_{j} x_{k+j}=g^{(k)}\left(s_{0}\right) \quad \text { for } \quad 0 \leq k<n
$$


in the unknowns $x_{0}, x_{1}, \ldots, x_{n+J-1}$. Since this system consists of $n$ equations and $n+J$ variables, it follows that the space of solutions has a positive dimension.

We claim that, amongst those infinitely many solutions, we can find a solution such that $x_{0} \neq 0$. In fact, if $\lambda_{0}=0$ this is clear. If $\lambda_{0} \neq 0$ we have

$$
x_{0}=\frac{1}{\lambda_{0}}\left(g\left(s_{0}\right)-\left(\lambda_{1} x_{1}+\cdots+\lambda_{J} x_{J}\right)\right) .
$$

If all $\lambda_{j}=0(j \geq 1)$, then $x_{0}=\lambda_{0}^{-1} g\left(s_{0}\right) \neq 0$. If some $\lambda_{j}(j \geq 1)$ does not vanish, then let $c$ be any fixed non-zero real number. We can solve the system (14) with the additional condition $x_{0}=c$ because of the above positivity of the dimension. Hence the claim follows.

Now we use Theorem 3 to find a positive real number $\tau$ such that

$$
\left|\lambda_{j} \zeta^{(k+j)}\left(s_{0}+i \tau\right)-\lambda_{j} x_{k+j}\right|<\frac{\epsilon}{3(J+1)}
$$

for $0 \leq k+j<n+J$. Summing (15) up on $j=0,1, \ldots, J$ and combining with (14), we obtain (13). This corresponds to (4) in the proof of Theorem 4. The rest of the proof follows as in the previous section; the constant $C$ can be made effective by solving the system of linear equations explicitly. Thus the proof of Theorem 6 is complete.

In the above proof, the assumption $g\left(s_{0}\right) \neq 0$ is used only in the case when all $\lambda_{j}=0(j \geq 1)$. Therefore this assumption is actually not necessary unless all $\lambda_{j}=0(j \geq 1)$. However the latter case is that we treated in Theorem 4, and we have already seen that the condition $g\left(s_{0}\right) \neq 0$ can be removed in the sense of Corollary 5 . Therefore we can conclude

Corollary 7. The condition $g\left(s_{0}\right) \neq 0$ can be removed from the statement of Theorem 6.

Acknowledgements. The idea for this paper originates from a workshop held at the Department of Mathematics of Universidad Autónoma de Madrid in December 2005 which was financially supported by the 'Rámon y Cajal'-programme of the Spanish Ministry for Science and Education. The authors are very grateful for this support and the kind hospitality at UA Madrid. They are also indebted to the referee for many useful comments and suggestions; especially, corollaries were first pointed out by the referee. 


\section{References}

[1] R. Garunkštis, The effective universality theorem for the Riemann zeta function, in: Proceedings of the Session in Analytic Number Theory and Diophantine Equations, Bonner Math. Schriften 360, Univ. Bonn, Bonn, 2003, 21 pp.

[2] R. Garunkštis, On the Voronin's universality theorem for the Riemann zeta-function, Fiz. Mat. Fak. Moksl. Semin. Darb. 6 (2003), 29-33.

[3] P. M. Gauthier and R. ClouÂtre, Approximation by translates of Taylor polynomials of the Riemann zeta function, Comput. Methods Funct. Theory 8(1-2) (2008), 15-19.

[4] A. Good, On the distribution of the values of Riemann's zeta function, Acta Arith. 38(4) (1980/81), 347-388.

[5] A. A. Karatsuba And S. M. Voronin, "The Riemann zetafunction", Translated from the Russian by Neal Koblitz, de Gruyter Expositions in Mathematics 5, Walter de Gruyter \& Co., Berlin, 1992.

[6] A. LAurinčIKas, A remark on the universality of the Riemann zeta-function, in: "Proceedings of XXXVIII Conf. of Lith. Math. Soc.", Vilnius, Technika, 1997, pp. 29-32.

[7] A. LAURINČIKAS, Effectivization of the universality theorem for the Lerch zeta function, (Russian), Liet. Mat. Rink. 40(2) (2000), 172-178; English translation in Lithuanian Math. J. 40(2) (2000), $135-139$.

[8] A. LAurinčIKas, Prehistory of the Voronin universality theorem, Šiauliai Math. Semin. 1(9) (2006), 41-53.

[9] K. Matsumoto, An introduction to the value-distribution theory of zeta-functions, Šiauliai Math. Semin. 1(9) (2006), 61-83.

[10] H. L. Montgomery, Extreme values of the Riemann zeta function, Comment. Math. Helv. 52(4) (1977), 511-518.

[11] D. V. PEČERsKIĬ, Rearrangements of the terms in function series, (Russian), Dokl. Akad. Nauk SSSR 209 (1973), 1285-1287; English translation in: Soviet Math. Dokl. 14 (1973), 633-636.

[12] J. Steuding, Upper bounds for the density of universality, Acta Arith. 107(2) (2003), 195-202.

[13] J. Steuding, Upper bounds for the density of universality. II, Acta Math. Univ. Ostrav. 13(1) (2005), 73-82.

[14] J. Steuding, "Value-distribution of L-functions", Lecture Notes in Mathematics 1877, Springer, Berlin, 2007. 
[15] S. M. Voronin, The distribution of the nonzero values of the Riemann $\zeta$-function, (Russian), Collection of articles dedicated to Academician Ivan Matveevič Vinogradov on his eightieth birthday. II, Trudy Mat. Inst. Steklov. 128 (1972), 131-150, 260.

[16] S. M. Voronin, The functional independence of Dirichlet $L$-functions, (Russian), Collection of articles in memory of Juriu Vladimirovič Linnik, Acta Arith. 27 (1975), 493-503.

[17] S. M. Voronin, A theorem on the "universality" of the Riemann zeta-function, (Russian), Izv. Akad. Nauk SSSR Ser. Mat. 39(3) (1975), 475-486, 703; English translation in: Math. USSR-Izv. 9(3) (1975), 443-453.

[18] S. M. Voronin, $\Omega$-theorems of the theory of the Riemann zetafunction, (Russian), Izv. Akad. Nauk SSSR Ser. Mat. 52(2) (1988), 424-436, 448; English translation in: Math. USSR-Izv. 32(2) (1989), 429-442.

Ramūnas Garunkštis and Antanas Laurinčikas:

Faculty of Mathematics and Informatics

Vilnius University

Naugarduko 24

03225 Vilnius

Lithuania

E-mail address: ramunas.garunkstis@maf .vu.lt

E-mail address: antanas.laurincikas@maf.vu.lt

Kohji Matsumoto:

Graduate School of Mathematics

Nagoya University

Furocho, Chikusaku

Nagoya 464-8602

Japan

E-mail address: kohjimat@math.nagoya-u.ac.jp

Jörn and Rasa Steuding:

Department of Mathematics

Würzburg University

Am Hubland, 97

218 Würzburg

Germany

E-mail address: steuding@mathematik.uni-wuerzburg.de

Primera versió rebuda el 6 de novembre de 2008, darrera versió rebuda el 31 de març de 2009. 